\title{
Adsorption induced transitions in soft porous crystals: An osmotic potential approach to multistability and intermediate structures
}

\author{
David Bousquet, ${ }^{1}$ François-Xavier Coudert, ${ }^{2}$ Alexandre G. J. Fossati, ${ }^{1}$ \\ Alexander V. Neimark, ${ }^{3}$ Alain H. Fuchs, ${ }^{2}$ and Anne Boutin ${ }^{1, a)}$ \\ ${ }^{1}$ CNRS-ENS-UPMC, Département de Chimie, École Normale Supérieure, 24 rue Lhomond, \\ 75005 Paris, France \\ ${ }^{2}$ CNRS \& Chimie ParisTech, 11 rue Pierre et Marie Curie, 75005 Paris, France \\ ${ }^{3}$ Department of Chemical and Biochemical Engineering, Rutgers University, Piscataway, \\ New Jersey 08854, USA
}

(Received 19 February 2013; accepted 11 April 2013; published online 3 May 2013)

\begin{abstract}
Soft porous crystals are flexible metal-organic frameworks that respond to physical stimuli (temperature, pressure, and gas adsorption) by large changes in their structure and unit cell volume. We propose here a thermodynamic treatment, based on the osmotic ensemble, of the interplay between guest adsorption and host deformation, where the bare host material can undergo elastic deformation, as well as structural transitions between metastable phases in the case of a multistable material. We show that in addition to structural transitions between metastable phases of bistable or multistable host frameworks, a new guest-stabilized host phase can be created when the size of the adsorbate is larger than the empty material's pore size. We then confront the findings of our approach with experimental data for systems exhibiting phenomena such as gate opening and breathing. () 2013 AIP Publishing LLC. [http://dx.doi.org/10.1063/1.4802888]
\end{abstract}

\section{INTRODUCTION}

Among the wide class of Metal-Organic Frameworks (MOFs) and other open framework hybrid organic-inorganic materials, the subclass of Soft Porous Crystals (SPCs) has attracted a lot of attention in the past few years. According to Kitagawa, who introduced this notion, ${ }^{1}$ "soft porous crystals are defined as porous solids that possess both a highly ordered network and structural transformability. They are bistable or multistable crystalline materials with long range structural ordering, a reversible transformability between states and permanent porosity." It has been recently shown that the "softness" of these materials, which was somewhat ill-defined up to now, is associated with a highly anisotropic elastic behavior, with some deformation directions exhibiting very low Young's and shear moduli. ${ }^{2}$ SPCs exhibit large-amplitude structural deformations induced by various external stimuli such as temperature, mechanical pressure, or guest adsorption. Guest-induced structural transitions include the "gate adsorption" and the "breathing" phenomena. Gate adsorption typically involves an abrupt structural transition between a nonporous structure and a porous host that is induced by gas adsorption. ${ }^{3,4}$ The breathing phenomenon consists of two successive adsorption-induced crystal-to-crystal transformations, from a large pore (lp) state to a narrow pore (np) state, and back again to the Ip structure. ${ }^{5,6}$

In a series of articles, the present authors have investigated the equilibrium thermodynamics of adsorption-induced and pressure-induced breathing transitions using an analytical approach based on the osmotic statistical ensemble.

\footnotetext{
a)Electronic mail: anne.boutin@ens.fr
}

Some key questions were answered, such as the prediction of the occurrence or absence of guest-induced breathing for a given guest-host system, ${ }^{7-9}$ and the successful derivation of a temperature-gas pressure phase diagram for the several gases (xenon, methane, carbon dioxide) in MIL-53 systems, ${ }^{10-12}$ as well as breathing phase diagrams for binary mixtures of methane and carbon dioxide in MIL-53. ${ }^{9,13}$ The physical mechanism of the breathing transitions was addressed by considering the adsorption-induced stress exerted on the material as a stimulus that triggers breathing transitions. The proposed model implies that the structural transitions in SPCs takes place when the stress reaches a certain critical threshold. ${ }^{14,15}$ This enabled to explain, at least qualitatively, the hysteresis phenomenon observed during breathing transitions. Finally, a multiscale dynamic model was developed in order to capture the dynamics of adsorption-induced deformation and structural transformation in MIL-53 based on the coupling of host-guest adsorption interactions with elastic response of the three-dimensional framework of a given geometry. ${ }^{16,17}$

On the molecular simulation side, Ghoufi and co-workers developed a forcefield that enabled to reproduce the $\mathrm{CO}_{2}$ adsorption-induced breathing transitions in MIL-53(Cr), ${ }^{18-20}$ as well as, more recently, the pressure-induced structural contraction in the same material, ${ }^{21}$ using hybrid Monte Carlo and osmotic MD simulations. A review of the analytical and direct simulations approaches can be found in Ref. 22.

Miyahara and co-workers investigated the gate adsorption phenomenon of a model porous coordination polymer which consisted of two mutually interpenetrating frameworks. ${ }^{23}$ They conducted a free energy analysis along a one-dimensional path that would lead to the pore opening and found a stable minimum along this path. In a more recent 
work, a 3D analysis of the same system revealed the existence of a second stable open structure. ${ }^{24}$ The evolution of the global free energy minimum with the external gas pressure was studied, and steps were predicted on the adsorption isotherms that were linked to structural transition of the host system. The present authors then proposed an extension of the Wang-Landau Monte Carlo algorithm to calculate the free energy surfaces in a similar manner to the work of Miyahara. ${ }^{25}$

Despite the progress made in understanding the guestinduced transitions in SPCs, some important issues remain to be addressed. In several guest-host systems, intermediate structures were observed upon gas adsorption, and it was not always clear whether or not they corresponded to stable structures/phases of the bare host material. This was the case for alkane adsorption in MIL-53(Fe), in which an intermediate triclinic structure was observed in addition to the already known narrow pore (np) and large pore (lp) forms. ${ }^{26}$ Following this observation, Ghoufi et al. suggested, from detailed MD simulations, that several metastable states could exist in MIL-53 materials, thus questioning their usually admitted bistability. ${ }^{20}$ Multistep nitrogen adsorption was also observed in $\mathrm{Co}(1,4$-benzenedipyrazolate) which was shown to correspond to transitions involving five different structures (i.e., three intermediate structures between the dry and the filled state). ${ }^{27,28}$ More recently, multistep gas adsorption was observed in ZIF-8 material. It involves at least two stable states of the empty material as well as some rearrangements of the guest molecules within the pore volume..$^{29,30}$

In order to address this multistability issue, we investigate here the full thermodynamic phase behavior of model guest-host systems, using a free energy landscape approach similar in nature to the one that Miyahara and co-workers suggested for the gate-opening transition. ${ }^{23,24}$ Depending on the characteristics of adsorption (evolution of Henry's constant, saturation uptake) and host deformation (whether the host is multistable or not), we describe phenomena such as gate opening and the existence of a possible intermediate structure in breathing materials.

\section{INTERPLAY OF ADSORPTION AND HOST FLEXIBILITY IN POROUS SOLIDS}

\section{A. Model of adsorption in flexible porous solids}

In the model proposed here, we consider the interplay between guest adsorption and host flexibility. In order to characterize the deformation of the host material, we depart from our earlier work and follow the approach of Watanabe et al. ${ }^{23}$ using a continuously-varying order parameter $\alpha$. For structural deformations to be coupled with adsorption, it is necessary that these deformations induce changes in the material's pore size (or pore volume). However, depending on the nature of the deformation modes, it may or may not affect the unit cell volume $V$ of the crystal. For example, in the case of "breathing" and "swelling" frameworks (Figure 1), the changes in unit cell angle or parameter are linked to cell volume variations. On the other hand, network flexibility in the form of either subnetwork displacement between interpenetrated frameworks, or linker side chain displacement, can happen at constant unit cell volume.

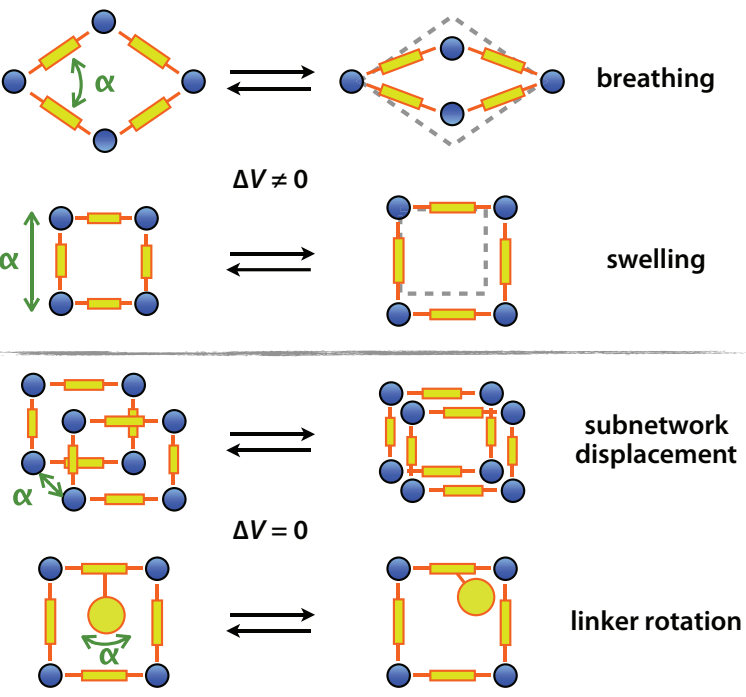

FIG. 1. Schematics of different modes of flexibility in soft porous crystals: the upper two involved a change in unit cell volume, while the lower two do not. In all cases, a continuous order parameter characterizing the deformation is indicated as $\alpha$.

The appropriate thermodynamic ensemble for the treatment of adsorption in flexible porous materials is the osmotic statistical ensemble $\left(N_{\text {host }}, \mu_{\text {ads }}, \sigma, T\right)$, where $N_{\text {host }}$ is the number of host unit cells, $\mu_{\mathrm{ads}}$ is the adsorbate chemical potential, $T$ is the temperature, and $\sigma$ is the tensorial mechanical stress exerted on the system boundaries. This is similar to the Grand Isostress ensemble used to describe surface force apparatus experiments. ${ }^{31}$ In most adsorption and isostatic compression experiments, this tensorial stress reduces to the scalar pressure $P$. Furthermore, in singlecomponent adsorption experiments, the mechanical pressure is related to the chemical potential of the external fluid by the $\mu(P)$ relation for the bulk fluid. Under these assumptions, we can write the free energy of the system as a function of the order parameter $\alpha$, which decomposes as proposed in our earlier work, ${ }^{7}$

$$
\begin{aligned}
\Omega(\alpha, P, T)= & F_{\text {host }}(\alpha, T)+P V_{\text {host }}(\alpha) \\
& -R T \int_{0}^{P} N_{\text {ads }}(\alpha, p, T) V_{\mathrm{m}}(p, T) \mathrm{d} p,
\end{aligned}
$$

where $V_{\mathrm{m}}(p, T)$ is the molar volume of the bulk fluid, $N_{\text {ads }}(\alpha, p, T)$ is the "fictitious" adsorption isotherm in a rigid material at fixed value of order parameter $\alpha$, and $F_{\text {host }}(\alpha, T)$ is the free energy of the material as a function of deformation.

In order to develop further a phenomenological model of adsorption-induced deformation in soft porous crystals, we need to make an assumption on the nature of the adsorption isotherms $N_{\text {ads }}(\alpha, p, T)$. It has been experimentally observed that the gas phase adsorption of small molecules in rigid MOFs overwhelmingly follows smooth type I isotherms, ${ }^{32}$ and that the same is true piecewise for stepped adsorption isotherms in bistable materials. ${ }^{7}$ We have used that fact to our advantage in a series of earlier models, dealing both with adsorption of single components ${ }^{10}$ and mixtures, ${ }^{9}$ and obtained good agreement with experimental data. ${ }^{13}$ We propose to integrate this approach in our continuous deformation model, 
by describing the adsorption isotherms with the Langmuir equation:

$$
N_{\mathrm{ads}}(\alpha, p, T)=\frac{K_{\mathrm{H}}(\alpha, T) P}{1+\frac{K_{\mathrm{H}}(\alpha, T) P}{N_{\max }(\alpha, T)}}
$$

in which the saturation uptake $N_{\max }$ and Henry's constant $K_{\mathrm{H}}$ both depend on deformation $\alpha$ as well as temperature. This introduces a natural coupling of guest adsorption and host deformation.

The model presented here aims at describing adsorption in flexible porous solids at thermodynamic equilibrium, by focusing on the local minima of the osmotic potential and their relative free energies. Adsorption experiments in soft porous crystals frequently show hysteretic phenomena, and the equilibrium transition is not directly observed and can only be bracketed by the adsorption and desorption transitions. We have shown in earlier work how such hysteresis can be interpreted either from a mechanical point of view (stress-based model $)^{14}$ or from the barriers in free energy profiles. ${ }^{25}$ The question of whether the hysteresis observed experimentally arise from one effect or the other, or a combination of both, has yet to be resolved.

\section{B. Model for the adsorption properties based on MIL-53 materials}

We have detailed in Sec. II A, the elements of a generic model that can be used to describe adsorption-induced continuous deformation of any porous solid. In this section, we showcase this generic approach on a model description of the MIL-53 family of "breathing" MOFs, allowing the determination of $K_{\mathrm{H}}(\alpha)$ and $N_{\max }(\alpha)$ at a given temperature. This model features lozenge-shaped pores with walls built of regularly spaced Lennard-Jones particles (more details of the unit cell setup and Lennard-Jones parameters are given in the supplementary material $\left.{ }^{33}\right)$. In this model, the order parameter of the deformation is the opening angle of the lozenge, $\alpha$ (cf. Fig. S1 of the supplementary material). ${ }^{33}$ We used Grand Canonical Monte Carlo (GCMC) simulations to describe the adsorption of guest Lennard-Jones particles in these pores, which reproduces well the experimental phenomenology of the MIL-53 breathing phenomenon, as showed in our earlier work. ${ }^{25}$ The GCMC isotherms obtained at a fixed temperature and for various values of $\alpha$ were fitted by the Langmuir equation to obtain profiles of $K_{\mathrm{H}}(\alpha)$ and $N_{\max }(\alpha)$ at a given temperature.

The profile of the Henry's constant (i.e., the gas-solid affinity in the low pressure limit) for the MIL-53 model is presented in Figure 2. It has the following features:

- In the limit of small opening angle $\alpha$, the material is very dense and the accessible pore size becomes smaller than particle size. No adsorption can take place, hence $K_{\mathrm{H}} \rightarrow 0$.

- In the limit of large opening angle, the accessible surface of the material becomes independent of $\alpha$ and $K_{\mathrm{H}}$ tends toward a constant non-zero value.

- Somewhere in-between, there exist a particular value of $\alpha$ for which the adsorbate fits exactly into the pore, interacting with multiple walls from the framework.

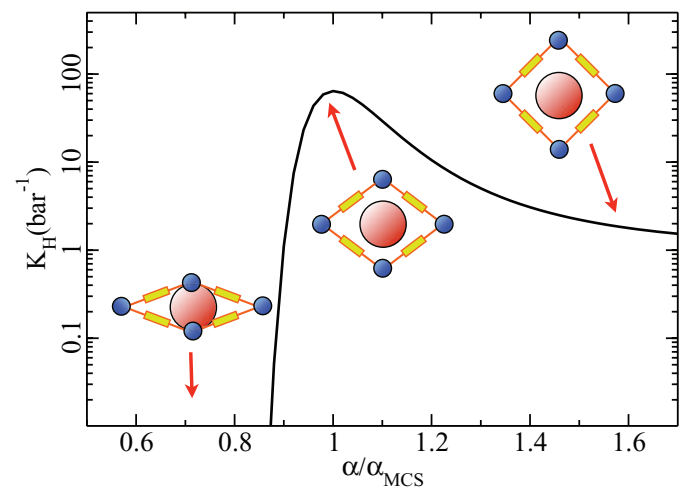

FIG. 2. Evolution of the Henry's constant, $K_{\mathrm{H}}$, in the lozenge-shaped pores of the MIL-53 model as a function of pore opening angle $\alpha$. Typical situations are schematized: $\alpha<\alpha_{\text {MCS }}$ (pore volume too small), $\alpha=\alpha_{\text {MCS }}$ (most comfortable structure), and $\alpha>\alpha_{\mathrm{MCS}}$ (large pore volume). The parameters for this Henry's constant profile are $K_{\mathrm{H}}=5.9 \times 10^{-8} \mathrm{bar}^{-1}, \alpha_{1}=23.57^{\circ}$, and $\alpha_{2}=15.52^{\circ}$ (see Eq. (3)).

This corresponds to a maximum of $K_{\mathrm{H}}(\alpha){ }^{34}$ We call this point of the curve the single particle most comfortable structure, or MCS, and denote the corresponding order parameter as $\alpha_{\mathrm{MCS}}$.

Because the physical considerations listed above are quite generic and should apply to most deformable porous structures, we expect that the Henry's constant profile, depicted in Figure 2 for the case of our MIL-53 model, is in fact a generic trait of these systems. To check this point, we calculated $K_{\mathrm{H}}$ for a pore system with different pore geometry: a slit pore with variable height $h$ (Fig. S2 of the supplementary material). ${ }^{33}$ The profile of the slit pore's Henry's constant $K_{\mathrm{H}}(h)$ (presented in Fig. S4 of the supplementary material) ${ }^{33}$ indeed shares the same characteristics.

We also characterized the evolution of adsorption saturation uptake $N_{\max }(\alpha)$, presented in Fig. S5 of the supplementary material. ${ }^{33}$ As expected, the saturation uptake increases with the pore size of the host, and thus with $\alpha$. In order to present a simple analytical model of the coupling between adsorption and deformation, we have fitted the profiles of both $K_{\mathrm{H}}(\alpha)$ and $N_{\max }(\alpha)$ by ad hoc analytical functions. $N_{\max }(\alpha)$ was fitted with a simple linear expression, $N_{\max }(\alpha)=A+B \alpha$ (we discuss this approximation in detail in Sec. IV). In order to fit the non-monotonic shape of $K_{\mathrm{H}}$, we used the form

$$
K_{\mathrm{H}}(\alpha)=K_{\mathrm{H}}^{0} \cdot \exp \left(\left(\frac{\alpha_{1}}{\alpha-\alpha_{2}}\right)^{8}-\left(\frac{\alpha_{1}}{\alpha-\alpha_{2}}\right)^{4}\right) .
$$

All fitting parameters are given in the legend of Figs. S5 and S6 of the supplementary material. ${ }^{33}$ The use of analytical functions rather than numerical interpolation makes the calculations below easier.

\section{Phenomenology of elastic deformation upon guest adsorption}

In this section, we provide a thermodynamic description of the interplay between adsorption and the simplest mode of solid deformation: the elastic deformation of the host 


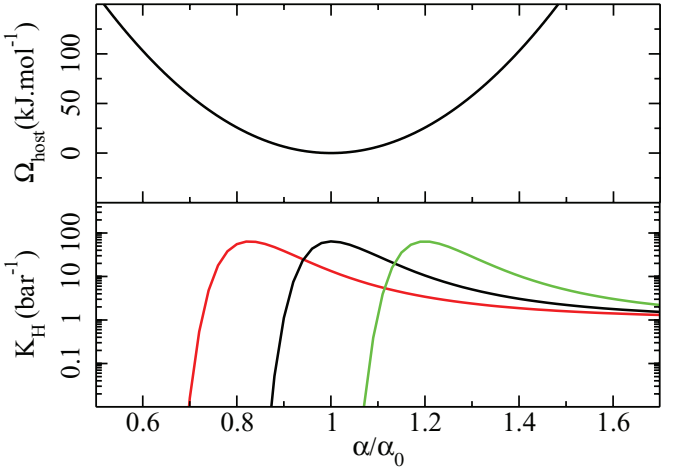

FIG. 3. Evolution of the Henry's constant as a function of host pore opening $\alpha$ for 3 different sizes of adsorbate (lower panel; $\alpha_{\mathrm{MCS}} / \alpha_{0}=0.8,1$, and 1.2, respectively), compared to elastic host free energy (upper panel). The free energy unit is $\mathrm{kJ} / \mathrm{mol}$ per unit cell.

framework around a "relaxed" structure corresponding to a local minimum in free energy. A development of the host free energy around this minimum will thus be of the form $F_{\text {host }}(\alpha)$ $=F_{0}+\frac{1}{2} K\left(\alpha-\alpha_{0}\right)^{2}$, where $F_{0}$ can be used as the reference free energy and set equal to zero (Fig. 3, upper panel). It is worth noting that $\alpha_{0}$ is the order parameter corresponding to the relaxed host structure, i.e., the most stable structure in the absence of adsorption. We then incorporated this expression of $F_{\text {host }}$ into the osmotic free energy (Eq. (1)), as well as the evolution of $K_{\mathrm{H}}(\alpha)$ and $N_{\text {max }}(\alpha)$ extracted from the MIL-53 adsorption model described in Sec. II B.

In order to study the effect of the adsorbed particle size, we have performed a series of calculation with various values of $\alpha_{\text {MCS }}$ by shifting the profiles of $K_{\mathrm{H}}(\alpha)$ (see Fig. 3) and scaling $N_{\max }(\alpha)$ inversely proportional to the particle volume (see supplementary material). ${ }^{33}$ We find that two very different cases arise depending on the relative values of the particle size and pore size, i.e., on the value of $\alpha_{\mathrm{MCS}} / \alpha_{0}$. We describe those two cases below.

Case 1: The first case, and probably the most common one, is that of an adsorbate size smaller than the pore size of the relaxed structure, leading to $\alpha_{\mathrm{MCS}}<\alpha_{0}$. The osmotic thermodynamic potential in such a case is plotted as the upper panel of Figure 4, for increasing values of the chemical potential $\mu$. At low pressures, the adsorption of the first guest molecules leads to a continuous contraction of the porous solid, bringing it closer to the most comfortable structure at the expense of a free energy penalty associated with the elastic deformation. At larger pressure the material will, on the other hand, exhibit swelling, as the host framework obviously requires to be expanded in order to accommodate more molecules. This nonmonotonic variation of the pore size (quantified by the order parameter $\alpha$, upper panel of Fig. 5) with increasing pressure leads to an isotherm that has a regular type I shape (Fig. 5, lower panel), even though the adsorption-induced deformations lead to deviation from a pure Langmuirian isotherm. This phenomenon has been previously reported in the literature, for both experimental and molecular simulation adsorption studies. In particular, such non-monotonic isotherms of solvation pressure have been calculated by using density functional theory ${ }^{34}$ and Monte Carlo

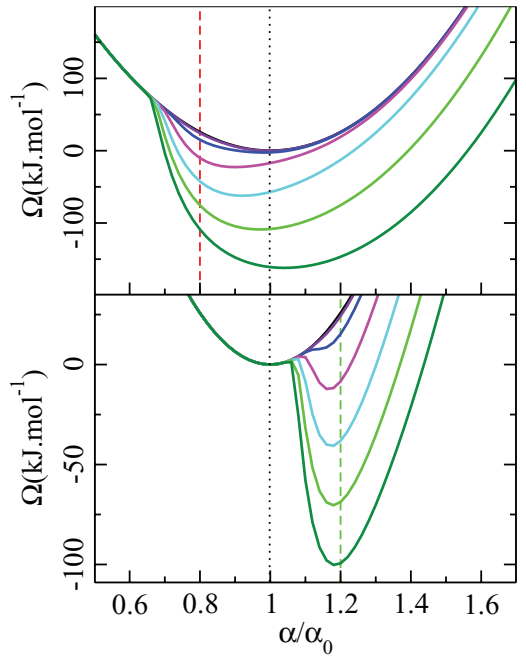

FIG. 4. Evolution of the profile of osmotic potential as a function of pore opening, for various values of external gas pressure. Top: case for a small adsorbate $\left(\alpha_{\mathrm{MCS}} / \alpha_{0}=0.8\right)$; bottom: case for a large adsorbate $\left(\alpha_{\mathrm{MCS}} / \alpha_{0}\right.$ $=1.2$ ).

simulations $^{35}$ to explain non-monotonic deformation in the course of adsorption on zeolites and activated carbons, which is typical for many microporous materials. ${ }^{36}$

Case 2: The second case is the adsorption of a larger molecule, for which the most comfortable structure would require a larger pore size than that of the relaxed porous solid $\left(\alpha_{\mathrm{MCS}}\right.$ larger than $\left.\alpha_{0}\right)$. In that case, the behavior of the \{host, guest $\}$ system is markedly different, and the material needs to expand to adsorb the first guest molecules. As seen in Fig. 5, this expansion will not happen gradually but the osmotic potential profile will instead present two minima (Fig. 4). The first minimum corresponds to the empty host material and the second, for a larger volume, to the expanded framework with adsorbed guest molecules (see also three-dimensional free energy profiles in Fig. S7 of the supplementary material ${ }^{33}$ ). The second state corresponds to the most comfortable structure, slightly deformed to minimize the free energy penalty due to elastic term. The existence of two metastable states upon guest adsorption, with

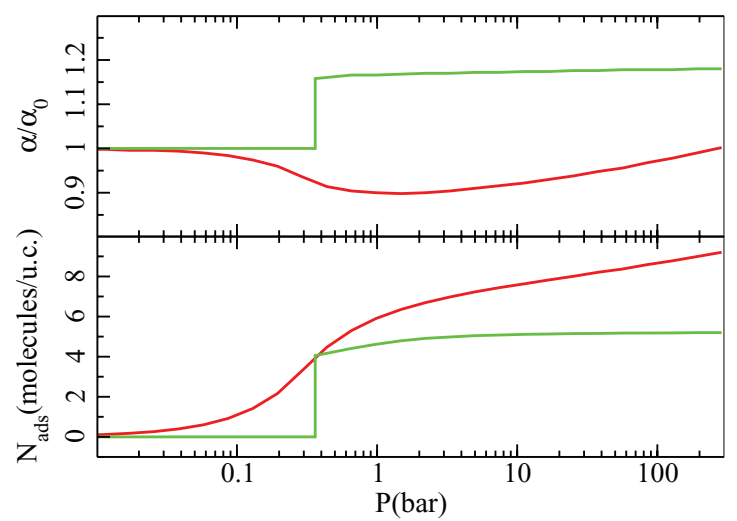

FIG. 5. Top: evolution of the pore opening angle of the host $\alpha$ as a function of external gas pressure for the cases discussed in Sec. II C: small adsorbate (red curve) and large adsorbate (green curve). Bottom: adsorption isotherms for both cases. 
the most comfortable structure becoming more stable as gas pressure increases, leads to a first-order structural transition between the empty structure and the MCS. This can be seen as a jump on the evolution of the material's deformation upon adsorption as well as a step on the adsorption isotherm (Figure 5).

Finally, we should mention that in between those two cases, it is also possible that the most comfortable structure is close to the relaxed structure: $\alpha_{0} \approx \alpha_{\mathrm{MCS}}$. Then, the behavior of the system during adsorption is similar to the continuous deformation demonstrated in the first case above. However, as the most comfortable structure is (almost) the relaxed state, the structure may not shrink and only undergoes a slight continuous expansion upon adsorption.

\section{Relevance for the "gate opening" phenomenon}

The stepwise structural transition described above in case $2\left(\alpha_{\mathrm{MCS}}>\alpha_{0}\right)$ is fully consistent with the experimental phenomenon known as adsorption-induced gate opening, which was first described in the case of interdigitated and interpenetrated frameworks whose pore volume depended on the presence of adsorbed guest molecules ${ }^{4,37}$ and later studied theoretically by the group of Miyahara. ${ }^{23,38}$ It consists of a transition, triggered by adsorption, from a nonporous structure into a more open form of the host framework, in the manner described in Sec. II C. In this section, we have used the experimental adsorption data of a series of gases on materials $\mathrm{Cu}\left(4,4^{\prime} \text {-bipy) (dhbc }\right)_{2}{ }^{4}$ and RPM3- $\mathrm{Zn}^{39}$ to validate this model. Since the gate opening effect happens when the solid's structure has to be significantly changed to accommodate guest molecules, the magnitude of the adsorption-induced structural deformation is expected to depend strongly on the size of the adsorbate. Hence, guest molecules of different size can be used as probes to evaluate the host free energy difference between the close empty state and the open adsorbing state: at the equilibrium pressure of gate opening transition, the adsorption part of the thermodynamic potential compensates exactly the host free energy penalty. We have calculated this size dependence for the two systems mentioned above, as adsorption-desorption isotherms had been published in the literature for a variety of different adsorbates in those materials. In $\mathrm{Cu}\left(4,4^{\prime} \text {-bipy)(dhbc) }\right)_{2}$, where $\mathrm{N}_{2}, \mathrm{CH}_{4}, \mathrm{O}_{2}$, and $\mathrm{CO}_{2}$ trigger the gate opening at room temperature and pressures of $\mathrm{P}=40 \pm 10$ bar, $\mathrm{P}=10 \pm 3$ bar, $\mathrm{P}=31 \pm 6$ bar, and $\mathrm{P}<2$ bar, respectively, we calculated the evolution of $\Delta F_{\text {host }}$ with the kinetic radius of the molecule following the procedure described by our group ${ }^{7}$ (see Table I). This trend goes in the right direction, as free energy is expected to increase with increasing deformations of the framework. However, because the adsorbates for which experimental data is available have similar sizes, the variation is not fully outside the uncertainty. The data for adsorption of $\mathrm{CH}_{4}, \mathrm{C}_{2} \mathrm{H}_{6}$, $\mathrm{C}_{3} \mathrm{H}_{8}$, and $\mathrm{C}_{4} \mathrm{H}_{10}$ in material RPM3-Zn, however, demonstrates a statistically significant dependence of $\Delta F_{\text {host }}$ on the length of the alkane chain adsorbed. This is in agreement with the description of the gate opening presented by our model.
TABLE I. Free energy difference between the empty material state and the most comfortable structure of different adsorbates in two gate opening systems: $\mathrm{Cu}\left(4,4^{\prime}\right.$-bipy $)(\mathrm{dhbc})_{2}{ }^{4}$ and RPM3-Zn. ${ }^{39}$

\begin{tabular}{|c|c|c|c|c|c|}
\hline \multicolumn{3}{|c|}{$\mathrm{Cu}\left(4,4^{\prime}\right.$-bipy $)(\mathrm{dhbc})_{2}$} & \multicolumn{3}{|c|}{ RPM3-Zn } \\
\hline & Radius (§) & $\Delta F_{\text {host }}(\mathrm{kJ} / \mathrm{mol})$ & & Radius $(\AA)$ & $\Delta F_{\text {host }}(\mathrm{kJ} / \mathrm{mol})$ \\
\hline $\mathrm{CO}_{2}$ & 2.8 & $\ldots$ & $\mathrm{CH}_{4}$ & 4.0 & $\ldots$ \\
\hline $\mathrm{O}_{2}$ & 2.8 & $3.9 \pm 0.6$ & $\mathrm{C}_{2} \mathrm{H}_{6}$ & 4.4 & $5.2 \pm 0.9$ \\
\hline $\mathrm{N}_{2}$ & 3.0 & $3.85 \pm 0.45$ & $\mathrm{C}_{3} \mathrm{H}_{8}$ & 4.9 & $6.6 \pm 1.7$ \\
\hline $\mathrm{CH}_{4}$ & 4.0 & $4.35 \pm 0.75$ & $\mathrm{C}_{4} \mathrm{H}_{10}$ & 5.1 & $13.7 \pm 4.7$ \\
\hline
\end{tabular}

Finally, it is interesting to note that the gate opening phenomenon has been observed in a large variety of systems. Some of the gate opening materials are formed by stacked 2D layers that can incorporate guest molecules between them upon increase of the unit cell volume. In other cases, such as interpenetrated or interdigitated frameworks, the gate opening phenomenon occurs at fixed unit cell volume, but with a displacement of the frameworks relative to one another, leading to an increase in pore size. Our model covers both cases with the same methodology, by relying on a physically meaningful order parameter to describe the deformation of the porous solid.

\section{THE CASE OF MULTISTABLE FRAMEWORKS}

\section{A. Predictions of the model}

We have now demonstrated that guest adsorption in an elastic host framework may involve either a gradual deformation of the framework, or a structural transition involving a phase of the material that is not stable in the absence of adsorbate (i.e., not a metastable structure of the empty host framework). However, there are known examples of so-called "multi-stable" materials, which may assume multiple metastable structures even in the absence of any guest molecule. An example of this is the MIL-53 family of metal-organic frameworks, ${ }^{6}$ formed by lozenge-shaped onedimensional pores, which exhibit structural transitions upon temperature variations ${ }^{40}$ or mechanical pressure. ${ }^{15}$ In this section, we apply our model to the case of a bistable material integrating into Eq. (1), an expression of the host free energy with two local minima (Fig. 6). ${ }^{16}$ For such a bistable material, we find that six different characteristic cases can be established depending on two factors: (i) which of the two structures is more stable in the absence of adsorption; (ii) the relative values of the particle size and the pore sizes (labelled 1,2 , and 3). The six possible cases explored are depicted on Fig. 6. Cases labelled A and B refer to the denser structure being more (respectively, less) stable than the open structure. The labels 1, 2, and 3 refer to the "most comfortable state" being smaller than the densest minimum, closest to the densest minimum but larger, and closest to the more open structure. The results obtained for all six cases are described in Figs. S10, S11, S12, S13, S14, and S15 of the supplementary material. ${ }^{33}$ While most of these cases correspond directly with our earlier models of adsorption-induced transitions in multistable materials, two of them correspond to phenomena that 

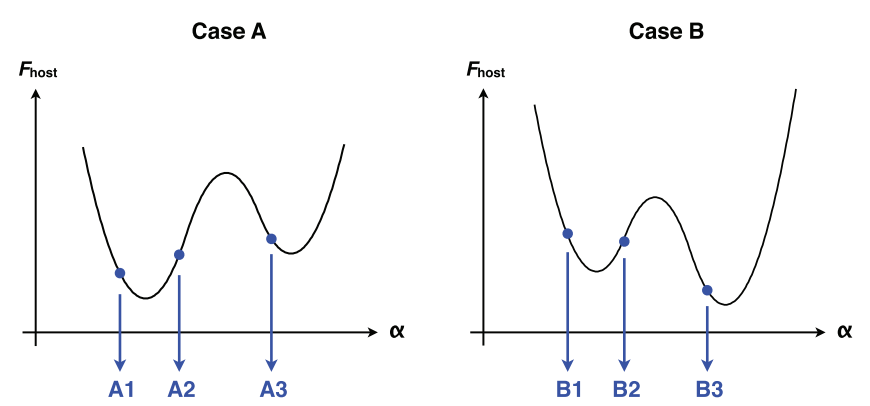

FIG. 6. Six characteristic cases of adsorption in a bistable host framework, depending on the free energy profile $F_{\text {host }}$ and the position of the most comfortable state for adsorption $\alpha_{\mathrm{MCS}}$, depicted by the blue dots for three different sizes of adsorbate molecules.

have not yet been proposed in the literature, and we describe them in details below.

Case A2: Let us first focus on the intriguing case where:

- the metastable state with a larger volume, which we will call the large pore (lp) state in keeping with established terminology of the MIL-53 literature, is less stable than the denser structure, which we will label the narrow pore (np) structure: $F_{\mathrm{np}}^{\text {host }}<F_{\mathrm{lp}}^{\text {host }}$;

- the most comfortable structure for adsorption falls inbetween the two states $\left(\alpha_{\mathrm{np}}^{0}<\alpha_{\mathrm{MCS}}<\alpha_{\mathrm{lp}}^{0}\right.$ ), in the free energy well of the np structure.

Under these assumptions, we used our model on a biparabolic free energy profile (shown in Fig. S8 of the supplementary material $)^{33}$ to calculate the full osmotic potential surface as a function of pore opening and gas pressure, which is depicted in Figure 7. On the profiles of osmotic potential, it can be seen that at very low pressure, the most stable state is the empty np state, until a first-order transition akin to gate opening occurs. The stable phase then becomes a deformed np state, close to the most comfortable structure, with adsorbed molecules: we label this intermediate structure int. At higher pressure, the material undergoes another structural transition into the Ip state, whose larger pore volume allows to accommodate more guest molecules, leading to its stability in the limit of high pressure (Fig. 8).

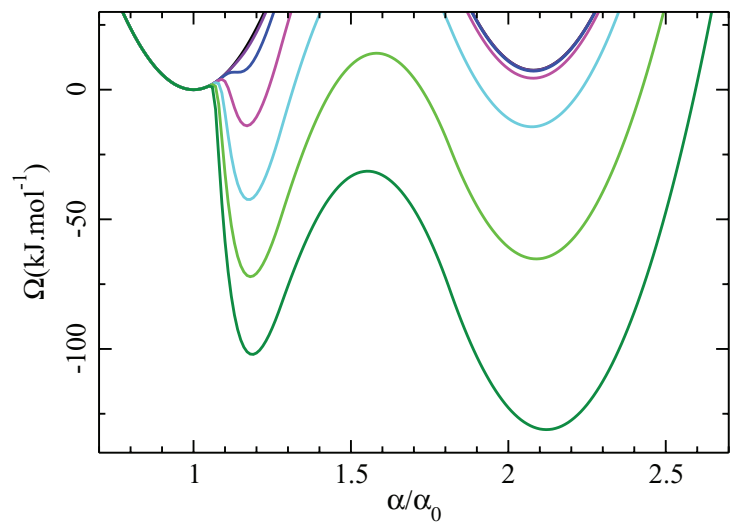

FIG. 7. Profile of osmotic potential upon adsorption for a bistable material, as a function of the channel opening, for different values of external gas pressure, in case A2 (see text). The reference $\alpha_{0}$ is taken to be the opening angle of the relaxed narrow-pore structure.

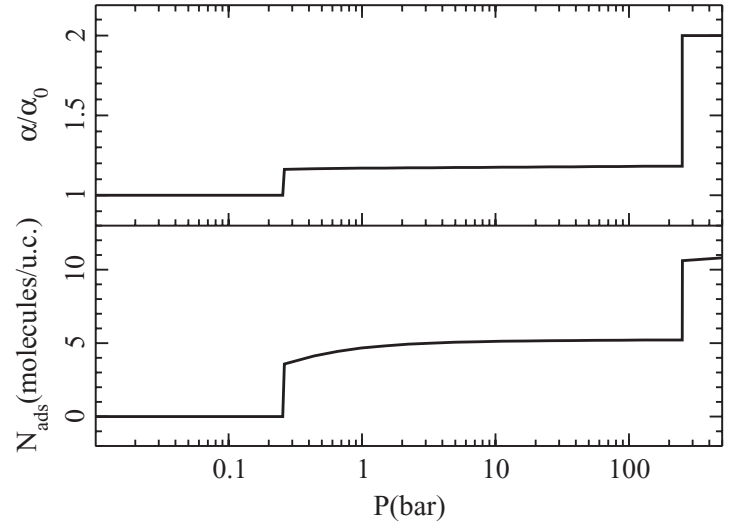

FIG. 8. Behavior of a bistable material upon gas adsorption, in case A2 (see text). Top: evolution of host pore opening as a function of external gas pressure; bottom: adsorption isotherm.

Case B2: We now turn to the symmetric case where the Ip state is intrinsically more stable than the np state in absence of guest molecules: $F_{\text {lp }}^{\text {host }}<F_{\text {np }}^{\text {host }}$. Upon adsorption, the material will start in the lp state, undergoing a contraction in the elastic regime to increase its host-guest interactions. At some pressure, a transition will take place into the denser int phase, whose larger Henry's constant makes it energetically more favorable. At higher loading, the material will undergo the reverse transition from int to $\mathbf{l p}$; this double structural transition upon adsorption is a form of "breathing": $\mathbf{l p} \rightarrow \mathbf{i n t} \rightarrow \mathbf{l p}$, as seen in Fig. S16 of the supplementary material ${ }^{33}$ (in case B1, one would observe the usual breathing involving the np phase: $\mathbf{l p} \rightarrow \mathbf{n p} \rightarrow \mathbf{l p}$ ). However, in case B2, the transition takes place between the Ip and int phases, while the metastable np structure is never observed upon adsorption. The reason is that $\mathbf{n p}$ is less stable than lp for the empty material, and its osmotic free energy is never lowered upon increase of gas pressure because its porous size is too small to accommodate guest molecules. We thus observe upon adsorption a double structural transition involving a structure different than the one observed in structural transitions involving temperature variations or mechanical constraints.

\section{B. Application to the experimental data on the MIL-53 family}

We now compare the results detailed above with experimental data on structures of the MIL-53 family, revealing the local flexibility that exists around both the narrow pore and the large pore form. An extensive list of crystalline structures have been reported experimentally for those materials depending on the nature of the metal, the adsorbate, the pressure, the temperature. These structures cover a large portion of the volume continuum. The experimental unit cell volumes reported in literature are shown in Figure 9 for materials of the MIL-53 family based on various metal (Al, Cr, Fe, Ga, $\mathrm{Sc}$, and V) either in the empty form or with different adsorbates. The adsorbates are sorted out according to their diameter, which is linked to their expected MCS volume. Of course, no single parameter can fully describe the size of 


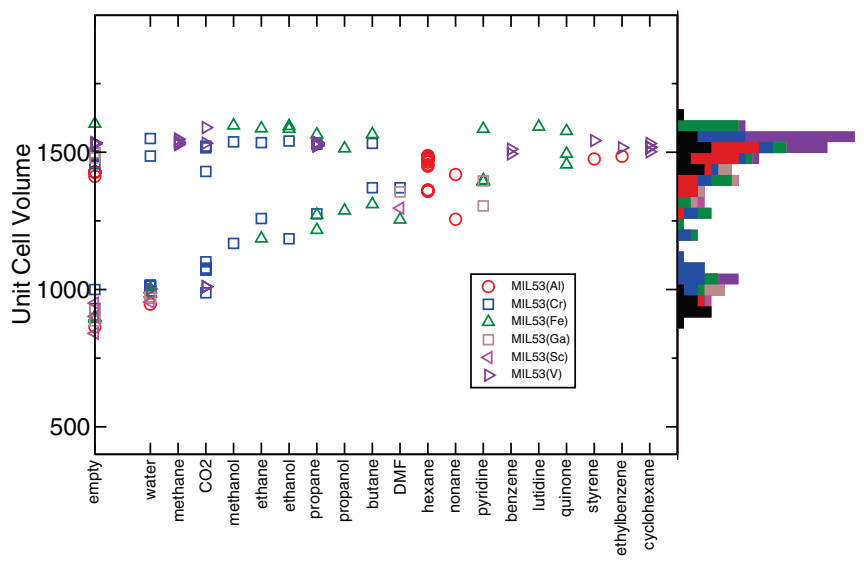

FIG. 9. Experimental values of the unit cell volume for materials of the MIL53 family with various metal centers and adsorbates, at various temperatures. Different metals correspond to different colors. The histogram of unit cell volumes is projected on the right side of the vertical axis with the same color key, except for empty materials which are all shown in black. ${ }^{6,40-66}$

nonspherical guest molecules and their ability to fit in the specific lozenge-shaped channels of the MIL-53 materials. Nonetheless, while the ordering of guest molecules in Figure 9 is somewhat arbitrary, the trend revealed is significant. The experimental volumes of the empty materials mainly lie either at $940-1000 \AA^{3}$ or $1400-1600 \AA^{3}$, corresponding to the narrow pore or large pore phases, respectively (see histogram in Fig. 9). With increasing adsorbate size, the volume of the narrow pore form increases while the volume of the large pore form is almost constant. For large adsorbates, only one form of the material is observed, with a unit cell volume corresponding to an open lp structure.

This behavior is fully coherent with our model. The evolution of the volume of the stable or metastable phase of our model in case B is reported in Figure 10 as a function of the opening angle of the most comfortable structure (which increases with adsorbate size). Since the volume in a given state slightly changes to accommodate guest molecules upon adsorption, the smaller and larger volumes observed for each phase in the pressure range of its stability domain are reported in Figure 10 as a filled color band.

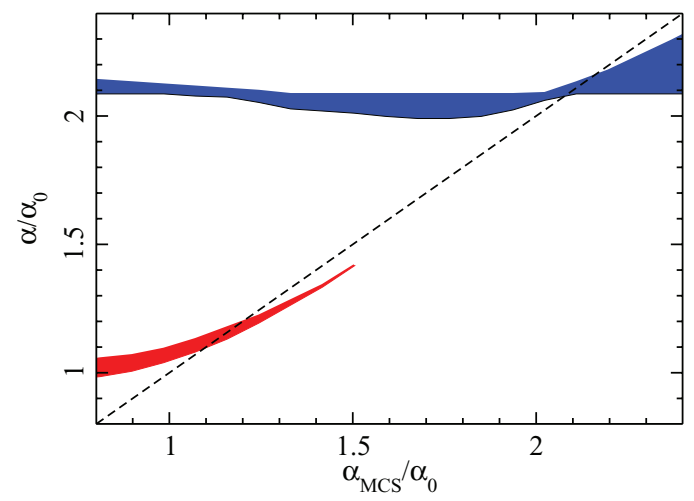

FIG. 10. Evolution of the pore opening angle of metastable states upon adsorption between 0 and 100 bar, as a function of the guest most comfortable structure $\left(\alpha_{\mathrm{MCS}} / \alpha_{0}\right)$. Domains corresponding to the narrow-pore and largepore structures are plotted in red and blue, respectively. The dashed line corresponds to a perfect fit of the adsorbate in the pores, i.e., $\alpha_{\mathrm{MCS}}=\alpha_{0}$.
In the case of very small adsorbates (small enough to fit in the empty np structure), the pore opening of both the np and Ip phases upon adsorption remains close to the values of the empty structures. For adsorbates of intermediate size $\left(0.8<\alpha_{\mathrm{MCS}} / \alpha_{0}<1.5\right)$, both phases deform in order to better fit the guest molecules, i.e., tend towards the most comfortable structure. This results in a slight contraction of the Ip phase and a dilatation of the np structure, the latter creating the so-called int phase. For large enough adsorbates, only the Ip phase is observed.

It is interesting to note that in the case of breathing, the local dynamics and the large structural transition are closely related. In the beginning of adsorption, the empty material is expected to be in the large pore form. Upon adsorption, the pore tends to reach the MCS, i.e., it shrinks slightly, up to the point where it reaches the first transition pressure and switch to the narrow pore form. The two phenomena have the same origin (converging toward the most comfortable structure), but simply have a different scale in magnitude. As more guest molecules get adsorbed in the narrow pore form, the material slightly expands to accommodate more molecules and reach the MCS. As some point, the second transition is reached, resulting in an open form of the material. Again, the two effects have the same causes, but consequences of a different amplitude.

The breathing phenomenon, coupled with elastic deformation of each phase, is triggered by the adsorption of small particles. But when adsorbed particles are larger, the adsorption-induced deformations are of a different kind. Specifically, whenever the guest is too large to fit in the small pore form of the material, the large pore form is the only stable state over the whole pressure range, and the small pore form is only an empty metastable state (corresponding to case B3). The adsorption-induced deformations switch from large breathing effect coupled to local guest-induced deformation to local contraction/dilation.

The phenomenology predicted by our model thus fully agree with experimental trend reported in Figure 9. It is worth mentioning that when comparing our predictions with experimental data obtained with different metal-based MIL53, we assume that the adsorption properties are mostly determined by the interaction with the ligands. Changing the metal would mostly influence the free energy profile of the empty host, and most notably the position of the minima and the elastic constants. These changes thus have a marked influence on the existence of the gate opening effect and the splitting of the small pores form into a np form and a int form. On the other hand, increasing the flexibility of the material will increase the stability domain of the int phase, thus decreasing the first transition pressure and increasing the second one.

It is worth noting that changes in temperature also strongly affect the free energy profile. ${ }^{10}$ Below (respectively above) the equilibrium Ip-np transition temperature, $T_{0}$, the stable phase is the np form and the system follows case A (respectively, case B) described in Sec. III A. While the value of the equilibrium temperature $T_{0}$ may obviously depends on the metal, no systematic work has yet been reported on that effect. 


\section{PACKING EFFECTS DURING THE PORE FILLING}

Results of the model presented so far were obtained assuming that the evolution of the adsorption saturation uptake in the flexible host, $N_{\max }(\alpha)$, varies linearly with the order parameter $\alpha$ that characterizes the deformation of the solid. In that case, we have shown that structural transitions can involve new structures, that are not metastable states of the empty host but exist because they are stabilized by strong host-guest interactions, i.e., variations of $K_{\mathrm{H}}(\alpha)$. However, there is another effect that can be considered: the packing effect. Depending on the geometry of the porous framework and on the nature of its deformations, the evolution of the saturation uptake $N_{\max }(\alpha)$ might not be linear or even regular, but instead correspond to a step-by-step filling of the pores. Indeed, in certain situations of very tight confinement, it is known that adsorption can involve several steps linked to reorganization between particularly stable configurations of the adsorbate. ${ }^{67}$ This can be seen, for example, on the profile of $N_{\max }(h)$ in the case of a slit pore with variable height $h$ (Figure S17 of the supplementary material ${ }^{33}$ ), which exhibits 3 clear steps. It can also be seen, although less markedly, on the evolution of $N_{\max }(\alpha)$ for our simple MIL-53 model (Fig. S5 of the supplementary material ${ }^{33}$ ).

In order to test the effect of step-by-step pore filling, we introduce an "artificial" step at $\alpha / \alpha_{0}=1.3$ in our previous linear approximation for $N_{\max }(\alpha)$ (see Figure S18 of the supplementary material ${ }^{33}$ ), while keeping the Henry's constant profile unchanged. The resulting profiles of osmotic potential, for the case of gate opening (case 2 in Sec. II C), are presented in Figure 11; we compare it to the similar situation of the bottom panel of Figure 4. In the later case (gate opening), we had two local minima of the free energy: the first one being the relaxed state of the empty host, and the second one close to the most comfortable single particle structure. We see here that the inclusion of a step in $N_{\max }(\alpha)$ leads to the appearance of a new metastable structure at $\alpha / \alpha_{0} \approx 1.3$, directly linked to the step-by-step nature of the adsorption. This additional metastable structure is involved in a first-order structural transition, and is reflected by an additional step in the adsorption isotherm (Figure 12). We thus conclude that more than one intermediate state can be generated by assuming a step-by-step

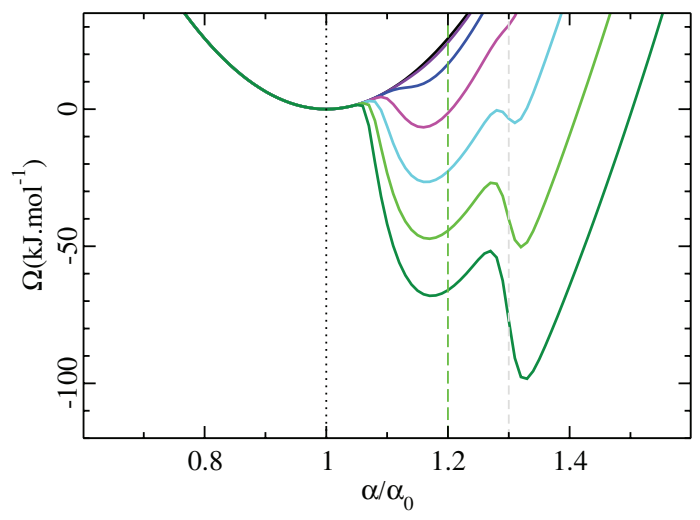

FIG. 11. Osmotic potential profile as a function of opening angle $\alpha$, for various values of the external gas pressure, in the case of an adsorbate with a step-by-step filling of the pores upon opening.

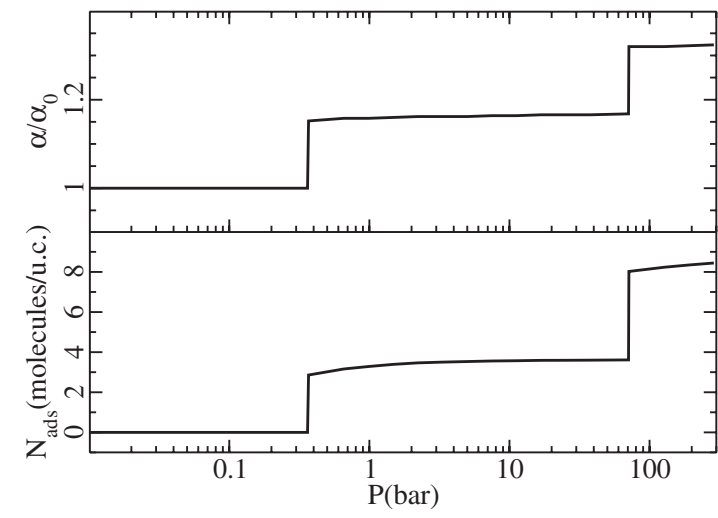

FIG. 12. Adsorption of a guest molecule showing step-by-step filling of the pores upon opening. Top: evolution of the pore opening angle of the host as a function of external gas pressure; bottom: adsorption isotherm.

filling of the pore volume upon adsorption, corresponding to some specific stable arrangements of the guest molecules for some given pore geometries. This might be the reason for the spectacular 5-step adsorption isotherm observed for $\mathrm{N}_{2}$ in CoBDP. ${ }^{28}$

\section{CONCLUSION}

We have investigated here the full thermodynamic phase behavior of guest-host systems in which the bare host material can undergo elastic deformation, as well as structural transitions between metastable phases in the case of a multistable material. Based on generic equations describing the free energy landscape for adsorption in a flexible host, we have calculated the osmotic grand potential as a function of guest-induced structural deformation using adsorption properties derived from a simple atomistic system that mimics the MIL-53 family of materials. In addition to structural transitions between metastable phases of bistable or multistable host frameworks, which have been amply described in the literature, we demonstrate that when the size of the adsorbate is larger than the empty material's pore size, a new gueststabilized host phase can be created. Such an observation is consistent with the experimental characteristics known for the gate opening phenomenon, as well as the existence of intermediate phases observed between narrow-pore and large-pore structures in the MIL-53 family. We demonstrated how stepby-step filling of the pore volume can lead to more than one intermediate state upon adsorption. The present approach is very general in that it only relies on two hypotheses, namely that the guest adsorption follows a type I isotherm and that the pore volume changes during the course of the host deformation. As an outcome of this work, we strongly suggest that the MIL-53 type framework (and possibly many of the SPCs that exhibit breathing transitions) is in itself a bistable crystal, and that whenever one or more extra intermediate structures are observed during the course of gas adsorption, they correspond to stable states of the guest-host system, and not to intrinsic stabilities of the empty host. 


\section{ACKNOWLEDGMENTS}

The Agence Nationale de la Recherche under project "SOFTCRYSTAB" (ANR-2010-BLAN-0822) is acknowledged for financial support.

${ }^{1}$ S. Horike, S. Shimomura, and S. Kitagawa, Nat. Chem. 1, 695 (2009).

${ }^{2}$ A. U. Ortiz, A. Boutin, A. H. Fuchs, and F.-X. Coudert, Phys. Rev. Lett. 109, 195502 (2012).

${ }^{3}$ D. Li and K. Kaneko, Chem. Phys. Lett. 335, 50 (2001).

${ }^{4}$ R. Kitaura, K. Seki, G. Akiyama, and S. Kitagawa, Angew. Chem., Int. Ed. 42, 428 (2003).

${ }^{5}$ G. Férey and C. Serre, Chem. Soc. Rev. 38, 1380 (2009).

${ }^{6}$ C. Serre, F. Millange, C. Thouvenot, M. Nogues, G. Marsolier, D. Louer, and G. Férey, J. Am. Chem. Soc. 124, 13519 (2002).

${ }^{7}$ F.-X. Coudert, M. Jeffroy, A. H. Fuchs, A. Boutin, and C. MellotDraznieks, J. Am. Chem. Soc. 130, 14294 (2008).

${ }^{8}$ F.-X. Coudert, C. Mellot-Draznieks, A. H. Fuchs, and A. Boutin, J. Am. Chem. Soc. 131, 3442 (2009).

${ }^{9}$ F.-X. Coudert, Phys. Chem. Chem. Phys. 12, 10904 (2010).

${ }^{10}$ A. Boutin, M.-A. Springuel-Huet, A. Nossov, A. Gedeon, T. Loiseau, C. Volkringer, G. Férey, F.-X. Coudert, and A. H. Fuchs, Angew. Chem., Int. Ed. 48, 8314 (2009)

${ }^{11}$ A. Boutin, S. Couck, F.-X. Coudert, P. Serra-Crespo, J. Gascon, F. Kapteijn, A. H. Fuchs, and J. F. M. Denayer, Microporous Mesoporous Mater. 140, 108 (2011).

${ }^{12}$ A. Boutin, F.-X. Coudert, M. A. Springuel-Huet, A. V. Neimark, G. Férey, and A. H. Fuchs, J. Phys. Chem. C 114, 22237 (2010).

${ }^{13}$ A. U. Ortiz, M. A. Springuel-Huet, F.-X. Coudert, A. H. Fuchs, and A. Boutin, Langmuir 28, 494 (2012).

${ }^{14}$ A. V. Neimark, F.-X. Coudert, A. Boutin, and A. H. Fuchs, J. Phys. Chem. Lett. 1, 445 (2010).

${ }^{15}$ A. V. Neimark, F.-X. Coudert, C. Triguero, A. Boutin, A. H. Fuchs, I. Beurroies, and R. Denoyel, Langmuir 27, 4734 (2011).

${ }^{16}$ C. Triguero, F.-X. Coudert, A. Boutin, A. H. Fuchs, and A. V. Neimark, J. Phys. Chem. Lett. 2, 2033 (2011).

${ }^{17}$ C. Triguero, F.-X. Coudert, A. Boutin, A. H. Fuchs, and A. V. Neimark, J. Chem. Phys. 137, 184702 (2012).

${ }^{18}$ F. Salles, A. Ghoufi, G. Maurin, R. Bell, C. Mellot-Draznieks, and G. Férey, Angew. Chem., Int. Ed. 47, 8487 (2008).

${ }^{19}$ A. Ghoufi and G. Maurin, J. Phys. Chem. C 114, 6496 (2010).

${ }^{20}$ A. Ghoufi, G. Maurin, and G. Férey, J. Phys. Chem. Lett. 1, 2810 (2010).

${ }^{21}$ P. G. Yot, Q. Ma, J. Haines, Q. Yang, A. Ghoufi, T. Devic, C. Serre, G. Férey, C. Zhong, and G. Maurin, Chem. Sci. 3, 1100 (2012).

${ }^{22}$ F.-X. Coudert, A. Boutin, M. Jeffroy, C. Mellot-Draznieks, and A. H. Fuchs, ChemPhysChem 12, 247 (2011).

${ }^{23}$ S. Watanabe, H. Sugiyama, H. Adachi, H. Tanaka, and M. Miyahara, J. Chem. Phys. 130, 164707 (2009).

${ }^{24} \mathrm{H}$. Sugiyama, S. Watanabe, H. Tanaka, and M. T. Miyahara, Langmuir 28, 5093 (2012)

${ }^{25}$ D. Bousquet, F.-X. Coudert, and A. Boutin, J. Chem. Phys. 137, 044118 (2012).

${ }^{26}$ P. L. Llewellyn, P. Horcajada, G. Maurin, T. Devic, N. Rosenbach, S. Bourrelly, C. Serre, D. Vincent, S. Loera-Serna, Y. Filinchuk, and G. Férey, J. Am. Chem. Soc. 131, 13002 (2009).

${ }^{27}$ H. J. Choi, M. Dinca, and J. R. Long, J. Am. Chem. Soc. 130, 7848 (2008).

${ }^{28}$ F. Salles, G. Maurin, C. Serre, P. L. Llewellyn, C. Knoefel, H. J. Choi, Y. Filinchuk, L. Oliviero, A. Vimont, J. R. Long, and G. Férey, J. Am. Chem. Soc. 132, 13782 (2010).

${ }^{29}$ D. Fairen-Jimenez, S. A. Moggach, M. T. Wharmby, S. Parsons, and T. Duren, J. Am. Chem. Soc. 133, 8900 (2011).

${ }^{30}$ C. O. Ania, E. Garcia-Perez, M. Haro, J. J. Gutierrez-Sevillano, T. ValdesSolis, J. B. Parra, and S. Calero, J. Phys. Chem. Lett. 3, 1159 (2012).

${ }^{31}$ P. Bordarier, B. Rousseau, and A. H. Fuchs, J. Chem. Phys. 106, 7295 (1997).

${ }^{32}$ K. S. W. Sing, D. H. Everett, R. A. Haul, L. Moscou, R. A. Pierotti, J. Rouquerol, and T. Siemieniewska, Pure Appl. Chem. 57, 603 (1985).

${ }^{33}$ See supplementary material at http://dx.doi.org/10.1063/1.4802888 for details on the simulated systems, force field, thermodynamic model, and free energy profiles.

${ }^{34}$ P. I. Ravikovitch and A. V. Neimark, Langmuir 22, 10864 (2006).

${ }^{35}$ P. Kowalczyk, A. Ciach, and A. V. Neimark, Langmuir 24, 6603 (2008).
${ }^{36}$ A. V. Tvardovskiy, Sorbent Deformation (Elsevier, 2006).

${ }^{37}$ D. Tanaka, K. Nakagawa, M. Higuchi, S. Horike, Y. Kubota, L. C. Kobayashi, M. Takata, and S. Kitagawa, Angew. Chem., Int. Ed. 47, 3914 (2008).

${ }^{38}$ S. Watanabe, H. Sugiyama, and M. Miyahara, Langmuir 24, 802 (2008).

${ }^{39}$ N. Nijem, H. Wu, P. Canepa, A. Marti, K. J. Balkus, Jr., T. Thonhauser, J. Li, and Y. J. Chabal, J. Am. Chem. Soc. 134, 15201 (2012).

${ }^{40}$ Y. Liu, J.-H. Her, A. Dailly, A. J. Ramirez-Cuesta, D. A. Neumann, and C. M. Brown, J. Am. Chem. Soc. 130, 11813 (2008).

${ }^{41}$ K. Barthelet, J. Marrot, D. Riou, and G. Férey, Angew. Chem., Int. Ed. 41, 281 (2002)

${ }^{42}$ V. Finsy, C. E. A. Kirschhock, G. Vedts, M. Maes, L. Alaerts, D. E. De Vos, G. V. Baron, and J. F. M. Denayer, Chem.-Eur. J. 15, 7724 (2009).

${ }^{43}$ R. I. Walton, A. S. Munn, N. Guillou, and F. Millange, Chem.-Eur. J. 17, 7069 (2011).

${ }^{44}$ F. Millange, N. Guillou, M. E. Medina, G. Férey, A. Carlin-Sinclair, K. M. Golden, and R. I. Walton, Chem. Mater. 22, 4237 (2010).

${ }^{45}$ G. de Combarieu, M. Morcrette, F. Millange, N. Guillou, J. Cabana, C. P. Grey, I. Margiolaki, G. Férey, and J. M. Tarascon, Chem. Mater. 21, 1602 (2009)

${ }^{46}$ C. Volkringer, T. Loiseau, N. Guillou, G. Férey, E. Elkaim, and A. Vimont, Dalton Trans. 12, 2241 (2009).

${ }^{47}$ Q. Ma, Q. Yang, A. Ghoufi, G. Férey, C. Zhong, and G. Maurin, Dalton Trans. 41, 3915 (2012).

${ }^{48}$ J. P. S. Mowat, V. R. Seymour, J. M. Griffin, S. P. Thompson, A. M. Z. Slawin, D. Fairen-Jimenez, T. Dueren, S. E. Ashbrook, and P. A. Wright, Dalton Trans. 41, 3937 (2012).

${ }^{49}$ M. Vougo-Zanda, J. Huang, E. Anokhina, X. Wang, and A. J. Jacobson, Inorg. Chem. 47, 11535 (2008).

${ }^{50}$ P. L. Llewellyn, G. Maurin, T. Devic, S. Loera-Serna, N. Rosenbach, C. Serre, S. Bourrelly, P. Horcajada, Y. Filinchuk, and G. Férey, J. Am. Chem. Soc. 130, 12808 (2008).

${ }^{51}$ X. Wang, J. Eckert, L. Liu, and A. J. Jacobson, Inorg. Chem. 50, 2028 (2011)

${ }^{52}$ T. K. Trung, P. Trens, N. Tanchoux, S. Bourrelly, P. L. Llewellyn, S. LoeraSerna, C. Serre, T. Loiseau, F. Fajula, and G. Férey, J. Am. Chem. Soc. 130, 16926 (2008).

${ }^{53}$ S. Bourrelly, B. Moulin, A. Rivera, G. Maurin, S. Devautour-Vino, C. Serre, T. Devic, P. Horcajada, A. Vimont, G. Clet, M. Daturi, J.-C. Lavalley, S. Loera-Serna, R. Denoyel, P. L. Llewellyn, and G. Férey, J. Am. Chem. Soc. 132, 9488 (2010).

${ }^{54}$ T. Devic, P. Horcajada, C. Serre, F. Salles, G. Maurin, B. Moulin, D. Heurtaux, G. Clet, A. Vimont, J.-M. Greneche, B. Le Ouay, F. Moreau, E. Magnier, Y. Filinchuk, J. Marrot, J.-C. Lavalley, M. Daturi, and G. Férey, J. Am. Chem. Soc. 132, 1127 (2010).

${ }^{55}$ G. Chaplais, A. Simon-Masseron, F. Porcher, C. Lecomte, D. Bazer-Bachi, N. Bats, and J. Patarin, Phys. Chem. Chem. Phys. 11, 5241 (2009).

${ }^{56}$ N. Rosenbach, Jr., A. Ghoufi, I. Deroche, P. L. Llewellyn, T. Devic, S. Bourrelly, C. Serre, G. Férey, and G. Maurin, Phys. Chem. Chem. Phys. 12, 6428 (2010).

${ }^{57}$ T. Whitfield, X. Wang, L. Liu, and A. Jacobson, Solid State Sci. 7, 1096 (2005).

${ }^{58}$ J. P. S. Mowat, S. R. Miller, A. M. Z. Slawin, V. R. Seymour, S. E. Ashbrook, and P. A. Wright, Microporous Mesoporous Mater. 142, 322 (2011).

${ }^{59}$ M. Maes, F. Vermoortele, L. Alaerts, S. Couck, C. E. A. Kirschhock, J. F. M. Denayer, and D. E. De Vos, J. Am. Chem. Soc. 132, 15277 (2010).

${ }^{60}$ A. Lyubchyk, I. A. A. C. Esteves, F. J. A. L. Cruz, and J. P. B. Mota, J. Phys. Chem. C 115, 20628 (2011).

${ }^{61}$ C. Serre, S. Bourrelly, A. Vimont, N. A. Ramsahye, G. Maurin, P. L. Llewellyn, M. Daturi, Y. Filinchuk, O. Leynaud, P. Barnes, and G. Férey, Adv. Mater. 19, 2246 (2007).

${ }^{62}$ P. L. Llewellyn, S. Bourrelly, C. Serre, Y. Filinchuk, and G. Férey, Angew. Chem., Int. Ed. 45, 7751 (2006)

${ }^{63}$ F. Millange, N. Guillou, R. I. Walton, J.-M. Greneche, I. Margiolaki, and G. Férey, Chem. Commun. 2008, 4732.

${ }^{64}$ N. Guillou, F. Millange, and R. I. Walton, Chem. Commun. 47, 713 (2011).

${ }^{65}$ T. Loiseau, C. Serre, C. Huguenard, G. Fink, F. Taulelle, M. Henry, T. Bataille, and G. Férey, Chem.-Eur. J. 10, 1373 (2004).

${ }^{66}$ H. Leclerc, T. Devic, S. Devautour-Vinot, P. Bazin, N. Audebrand, G. Férey, M. Daturi, A. Vimont, and G. Clet, J. Phys. Chem. C 115, 19828 (2011).

${ }^{67}$ R. Krishna, B. Smit, and S. Calero, Chem. Soc. Rev. 31, 185 (2002). 